\title{
Review
}

\section{Gallstones: an intestinal disease?}

During the first half of this century, cholelithiasis was regarded as a disease of the gall bladder, with inflammation of the wall as the primary defect and exfoliated cells as the source of biliary cholesterol. ${ }^{12}$ During the 1960s, Small and Rapo elucidated the physical-chemical basis of biliary cholesterol supersaturation and demonstrated that excessive hepatic cholesterol secretion is the underlying defect. ${ }^{3}$ Cholesterol gallstones were then considered to be the consequence of a metabolic defect of the liver. The importance of the gall bladder during gallstone formation was resurrected during the 1980 s, when impaired gall bladder emptying was identified as a factor contributing to gallstone formation. ${ }^{45}$ During the past 10 years, a third organ has been proposed as an important player in gallstone pathogenesis - the intestine. Several recent observations indicate slow intestinal transit in gallstone patients and animal models. Impaired intestinal transit might promote gallstone formation by increasing the amounts of the hydrophobic bile salt deoxycholate or by other mechanisms. In this review, we shall evaluate critically the pros and cons of the "intestinal hypothesis".

Cholesterol supersaturation and crystal formation Cholesterol is a relatively insoluble amphiphile. Its solubility in water is only $\sim 20 \times 10^{-9} \mathrm{M}$, but in gall bladder bile, $\sim 20 \times 10^{-3} \mathrm{M}$ of the sterol can be kept in solution. ${ }^{6}$ This millionfold increase in solubility is explained by incorporation of cholesterol into micelles, together with bile salts and phospholipids (mainly phosphatidylcholine). Whereas cholesterol is soluble only to a limited extent in micelles that contain exclusively bile salts ("simple micelles"), addition of phosphatidylcholine leads to strongly enhanced solubility in "mixed" micelles, provided that this phospholipid contains unsaturated acyl chains at the sn-2 position. ${ }^{7}$ Supersaturation occurs when either too much cholesterol or not enough bile salts and phosphatidylcholine are secreted to allow complete micellar solubilisation of all cholesterol in bile. Current thought is that increased biliary cholesterol secretion rather than decreased bile salt or phospholipid secretion is the primary cause of biliary cholesterol supersaturation. ${ }^{8}$ Excessive cholesterol may be solubilised in vesicles (spherical bilayers of cholesterol and phospholipids, without bile salts). As these vesicles are often cholesterol-rich, they may-possibly after aggregation and fusion - nucleate cholesterol crystals, an essential step in gallstone formation. ${ }^{9}{ }^{10} \mathrm{~A}$ large number of proteins are secreted in bile, among them apolipoproteins A1 and A2, anionic polypeptide fraction, aminopeptidase $\mathrm{N}, \alpha 1$ acid glycoprotein, mucin, haptoglobin, immune globulins, phospholipases A2 and C, and others. Based on in vitro studies, several of these proteins may either promote or inhibit crystallisation by stabilising or destabilising cholesterol-rich vesicles.

\section{Postprandial gall bladder motility}

Ingestion of a meal induces considerable gall bladder emptying, up to $70-80 \%$ of fasting gall bladder volume, by releasing the hormone cholecystokinin (CCK) from the duodenal wall. Apart from biliary cholesterol supersaturation and proteins, gall bladder motility may also influence gallstone formation. The basic concept is relatively simple: impaired gall bladder emptying prolongs residence of bile in the gall bladder, thus allowing more time for nucleation of cholesterol crystals from supersaturated bile. Furthermore, in the case of adequate gall bladder emptying, cholesterol crystals that have nucleated are ejected to the duodenum, whereas in the case of impaired gall bladder emptying, these crystals aggregate into macroscopic gallstones. Indeed, studies in patients with gallstones by either scintigraphic or ultrasonographic methods have identified a subgroup with impaired emptying, as defined by a low percentage decrease in fasting volumes or low amounts of ejected bile after consumption of a meal. ${ }^{4}$ Even in patients with normal gall bladder emptying according to these criteria, there are often increased fasting and residual gall bladder volumes, despite normal amounts of bile ejected after feeding. ${ }^{112}$ Traditionally, it has been assumed that after a meal, the gall bladder empties in a steady, progressive fashion and that gall bladder refill occurs only in the fasting state, between meals. This assumption has been challenged by studies using combined duodenal perfusion and scintigraphy ${ }^{13}$ or minute by minute ultrasound ${ }^{14}$ : there seems to be very frequent, short periods of gall bladder emtying and refilling ("bellow" concept), the balance between them determining whether net emptyingsuch as in the immediate postprandial period-or net refilling occurs. One should realise that, strictly speaking, ultrasound measures only gall bladder volumes (which are influenced by refilling), and HIDA scintigraphy measures the reduction in total gall bladder isotope counts (which are independent of refilling). Jazrawi and colleagues ${ }^{15}$ recently introduced a method of combined scintigraphy and ultrasound that enables simultaneous, accurate quantitation of gall bladder emptying and refilling, as well as bile turnover. Interestingly, postprandial gall bladder emptying, refilling and bile turnover were all strongly depressed in patients with gallstones, thus suggesting a reduced postprandial washout effect. Nevertheless, one may argue that abnormal gall bladder motility in these patients could be secondary to the presence of stones or supersaturated bile in the gall bladder, with no relevance for gallstone formation. Indeed, studies in gallstone forming animal models have shown that, after its absorption by the gall bladder wall from supersaturated bile, excess cholesterol is incorporated within the sarcolemnal plasma membrane of the gall bladder muscle cell, with decreased membrane fluidity, impaired contractility and impaired relaxation as a result. ${ }^{16-18}$ Although impaired motility could be in many cases secondary to biliary cholesterol supersaturation, it may still facilitate the process of gallstone formation: in the prairie dog model ${ }^{19}$ and in humans, ${ }^{20}$ impaired gall bladder motility occurs in the earliest stage of gallstone formation, before stones have formed. Gall bladder motility is also often impaired in many high risk situations for gallstone formation, such as pregnancy, obesity, diabetes mellitus, treatment with the somatostatin analogue octreotide, very low calory diets, and total parenteral nutrition. Prospective studies indicate that impaired gall bladder motility is also an independent risk factor for gallstone recurrence after extracorporeal shockwave lithotripsy. ${ }^{21}$ Daily CCK injection during total parenteral nutrition ${ }^{22}$ or

Abbreviations used in this paper: CCK, cholecystokinin; MMC, migrating motor complex. 


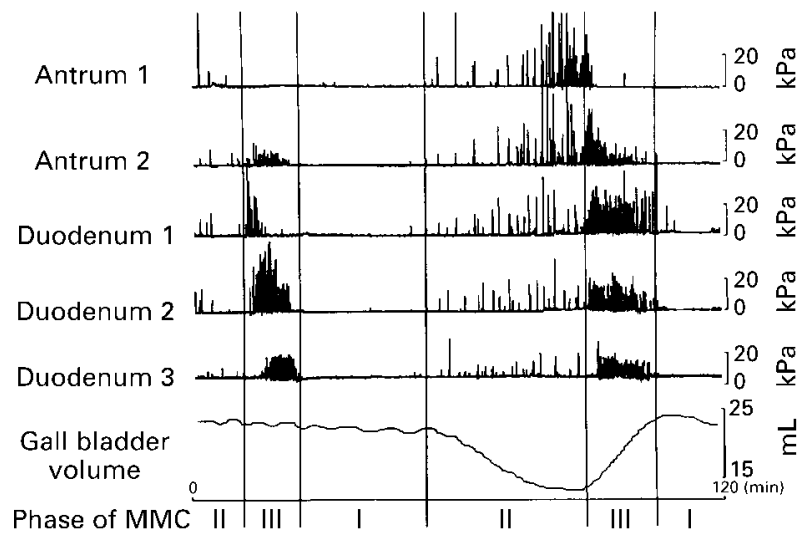

Figure 1 The intestinal migrating motor complex occurs periodically (1-2 hour cycle) during the fasting state and is characterised by three phases: contractile activity is absent during phase I; irregular activity occurs during phase II; and there are intense, regular coordinated contractions during phase III. Significant periodic gall bladder contraction occurs before phase III and is associated with a rise in plasma motilin concentrations.

inclusion of small amounts of fat in the diet during rapid weight $\operatorname{loss}^{23}$ restore normal gall bladder motility and avoid the risk of gallstone formation. All these data suggest a role for gall bladder motility in gallstone formation.

The intestine: its relation to bile composition and gall bladder motility in the fasting (interdigestive) state Recent data indicate that, along with the liver and the gall bladder, the intestine might also be involved in gallstone formation. The gall bladder and the small intestine determine enterohepatic cycling of bile salts and their flux through the liver. In patients with gallstones, both small intestinal ${ }^{24}$ and whole gut ${ }^{25}$ transit times are prolonged. What explanations could be offered for the association between prolonged intestinal transit and gallstone disease? One should remember one potential caveat: gallstones are rather frequent in the western world and the symptoms often attributed to them, such as right upper quadrant pain - with or without radiation to the back-or nausea, are not specific to gallstones. ${ }^{26}$ These symptoms can also be caused by constipation as they often disappear upon changes in diet or pharmacological intervention. Aspecific abdominal symptoms may have led to ultrasonographic detection of gallstones and clinicians with special interest in gallstone research may have measured intestinal transit and included these patients in their case-control studies. Nevertheless, one study explicitly states that patients were unaware of the presence of stones. ${ }^{25}$ One could also hypothesise that impaired intestinal motility is somehow causally related to gallstone formation. Indeed, the hydrophobic bile salt deoxycholate could be the link between impaired intestinal motility and lithogenic bile. The main bile salts in humans are cholate and chenodeoxycholate, both "primary" bile salts synthesised from cholesterol in the liver, and the "secondary" bile salt deoxycholate formed in the distal small intestine and colon by bacterial $7 \alpha$-dehydroxylation of cholate. Deoxycholate is partly absorbed from the intestinal lumen and joins the enterohepatic circulation of bile salts after taurine or glycine conjugation in the liver. Prolonged intestinal transit time probably enhances formation of deoxycholate by increasing the intestinal residence time of bile salts, whereas decreasing intestinal transit time may have the opposite effect. As a result amounts of deoxycholate vary considerably in humans, between 10 and $30 \%$ of the total bile salt pool. Preliminary data from Dowling and coworkers ${ }^{27}$ have revealed that patients with gallstones have increased amounts of Gram positive anaerobic bacteria and increased $7 \alpha$-dehydroxylating activity in their caecum compared with controls. In these patients, slowness of colonic transit correlates with increased caecal $\mathrm{pH}$ and water solubility of deoxycholate (factors promoting intestinal absorption of the bile salt). What effects do increased amounts of biliary deoxycholate in bile have on lithogenicity? Several studies in patients with gallstones have found a positive correlation between the amount of biliary deoxycholate on the one hand and the cholesterol saturation index ${ }^{24}$ or the speed of cholesterol crystallisation $^{28}$ on the other. In acromegalics, octreotide treatment induces a similar combination of increased intestinal transit times, more biliary deoxycholate, increased biliary cholesterol saturation index and fast crystallisation. ${ }^{29}{ }^{30}$ In contrast, treatment with ampicillin leads to decreased faecal $7 \alpha$-dehydroxylation activity, decreased amounts of biliary deoxycholate and a lower biliary cholesterol saturation index. ${ }^{31}$ In the ground squirrel ${ }^{32}$ and in the $\mathrm{C} 57 \mathrm{~L}$ inbred mouse, ${ }^{33}$ a cholesterol-rich diet induces a significant increase in the proportion of biliary deoxycholate, cholesterol supersaturation and gallstone formation. Furthermore, in the ground squirrel model, small intestinal transit is prolonged. ${ }^{32}$ Thus, data from patients with gallstones and acromegalics on octreotide as well as animal models suggest an association between slow intestinal transit, high deoxycholate, cholesterol supersaturation and gallstone formation. One should realise, however, that this association is not universal: in humans on oral contraceptives (another risk factor for gallstone formation), cholesterol supersaturation and slow intestinal transit are associated with a decreased rather than increased deoxycholic acid pool size. ${ }^{34}$ Furthermore, in both the ground squirrel and the $\mathrm{C} 57 \mathrm{~L}$ inbred mouse models on lithogenic diets, there is an increased proportion, not only of biliary deoxycholate, but also of the primary bile salt chenodeoxycholate. This might be explained by negative feedback suppression through the hydrophobic deoxycholate on hepatic cholesterol $7 \alpha-$ hydroxylase (the rate controlling enzyme in the classic pathway of bile salt synthesis) and a higher contribution to bile salt synthesis of the alternative or acidic pathway through the mitochondrial enzyme sterol $27 \alpha$-hydroxylase as the alternative pathway has a preference for chenodeoxycholate over cholate synthesis. ${ }^{35} 36$

Increased deoxycholate could promote lithogenic bile and gallstone formation through several mechanisms. Firstly, deoxycholate itself can slow down intestinal transit, $^{37}$ thereby allowing more time for intestinal cholesterol absorption and exerting a positive feedback on its own formation. The mechanism for this effect is not entirely clear: although in in vitro studies, bile salts seem to exert a depressant effect on intestinal muscle contractility, ${ }^{38}$ increased maximal contractile responses of intestinal circular and longitudinal smooth muscle strips on bethanechol stimulation have been reported in ground squirrels on a lithogenic diet, associated with slow small intestinal transit and biliary deoxycholate enrichment. ${ }^{32}$ More deoxycholate could theoretically also increase intestinal cholesterol absorption by means of enhanced micellar solubilisation of the sterol. This effect, however, is probably more relevant in animal models with rather hydrophilic endogenous bile salts ${ }^{32} 33$ than in humans with a relatively hydrophobic endogenous bile salt pool. Secondly, deoxycholate could enhance biliary cholesterol secretion by an effect on the hepatocytic canalicular membrane. The outer leaflet of the membrane contains large amounts of cholesterol and the phospholipid sphingomyelin (associated in laterally separated domains), which confers resistance to the detergent action of bile salts by decreasing membrane 
fluidity. Particularly hydrophobic bile salts such as deoxycholate can release cholesterol from the sphingomyelin domains (most likely by decreasing the activation energy necessary for desorption of the sterol from the membrane), thus allowing its secretion into bile. ${ }^{7}$ Thirdly, in vitro studies have revealed that deoxycholate enhances biliary cholesterol crystallisation by destabilising cholesterol-rich vesicles. ${ }^{39}$ This effect (paradoxical if one would only consider the enhanced micellar cholesterol solubilisation in deoxycholate-rich biles) can be nicely explained by the elegant model studies performed by Wang and Carey ${ }^{40}$ : deoxycholate leads to a rightward shift in the ternary (cholesterol-phospholipid-bile salt) phase diagram, with inherent faster cholesterol crystallisation.

Apart from the effects on biliary deoxycholate another, possibly complementary, link between the intestine and gallstone formation could be the relation between intestinal and gall bladder motility in the fasting (also called "interdigestive") state. As fig 1 shows, significant periodic gall bladder emptying occurs during this period (20-30\% emptying in the fasting state compared with $70-80 \%$ emptying after a meal) at one to two hour intervals, associated with the cycle of the intestinal migrating motor complex (MMC) and with a rise in plasma motilin concentrations..$^{41}$ The MMC, a pattern of cyclic contractile activity displayed in the fasting state by the upper intestinal tract, is characterised by three phases: during phase I, contractile activity is absent; irregular activity occurs during phase II; and during phase III there are intense, regular coordinated contractions. In healthy people, significant gall bladder emptying and high plasma motilin concentrations are observed before phase III. ${ }^{42-44} \mathrm{We}$ found that patients with gallstones have less frequent MMC cycles, absent interdigestive gall bladder emptying and an altered pattern of motilin release compared with controls. ${ }^{45}$ A similarly prolonged MMC cycle has been found in the ground squirrel model of gallstone formation. ${ }^{46}$ The fasting state, namely the night, would seem to be the most vulnerable period for gallstone formation. During this period, biliary cholesterol saturation is highest as a result of relatively low bile salt secretion and relatively high cholesterol secretion. There is also a progressive concentration of gall bladder bile during this period, which is partially counteracted by periodic interdigestive gall bladder contractions in association with phase III of the MMC. ${ }^{47}$ Bile concentration has important consequences: it leads to higher micellar cholesterol (and phospholipid) solubilisation by increasing the micellar phase boundary. ${ }^{40}$ As phospholipids are much more easily solubilised in micelles than cholesterol, there is a much larger shift of phospholipid than cholesterol from vesicles to micelles. The remaining vesicles should therefore be enriched in cholesterol and prone to nucleate cholesterol crystals. $^{48}{ }^{49}$ According to this concept, the reduced frequency of phase III and absent interdigestive gall bladder contractility, as found in our patients with gallstones, could promote bile concentration, crystallisation and gallstone formation. During the earliest stages of gallstone formation in the prairie dog model there is also excessive concentration of bile within the gall bladder ${ }^{50}$ and prevention of excessive bile concentration with the aid of the drug amiloride prevents gallstone formation. ${ }^{51}$ Interestingly, cholesterol absorption by the gall bladder wall seems to function as a protective mechanism against cholesterol crystallisation under these circumstances, because it leads to a lower biliary cholesterol saturation. Using the isolated pig gall bladder model, Corradini and colleagues ${ }^{52}$ have confirmed earlier reports ${ }^{53} 54$ that significant cholesterol absorption as well as some phospholipid absorption occurs from supersaturated biles. In contrast, there is virtually no bile salt absorption. This defence mechanism may be operating particularly in the case of impaired gall bladder emptying. ${ }^{55}$ Based on indirect

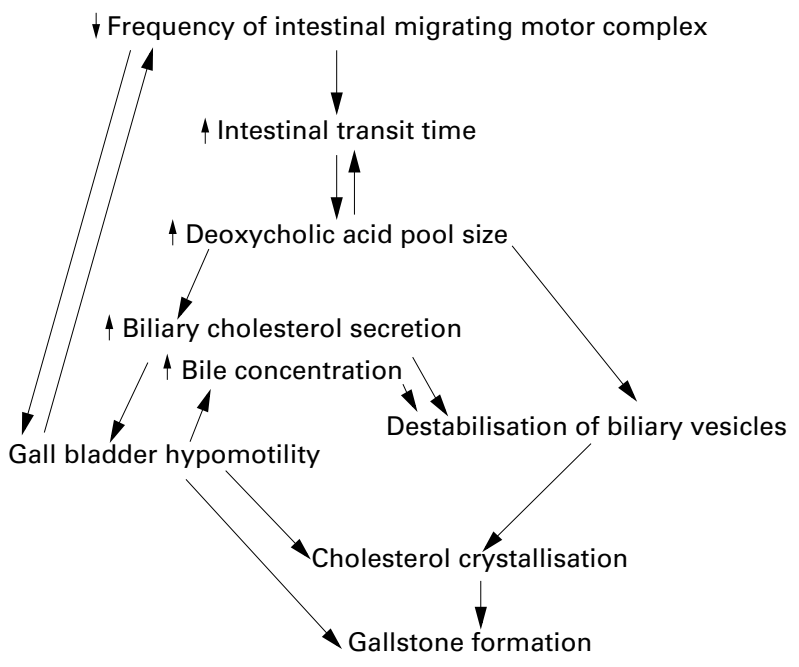

Figure 2 Potential mechanisms of cholesterol gallstone formation.

evidence (comparison of protein concentrations in hepatic and associated gall bladder biles), Groen and coworkers ${ }^{56}$ have postulated a similar absorption of pronucleating proteins by the gall bladder wall as an additional defence mechanism against crystallisation.

\section{Summary}

Current evidence suggests that impaired intestinal motility may facilitate gallstone formation by influencing biliary deoxycholate levels or by modulating interdigestive gall bladder motility (fig 2), although a primary intestinal defect in gallstone pathogenesis has not yet been demonstrated. In the cold war period, most interesting events, from a political point of view, occurred at the border between capitalist and communist systems, near the iron curtain. Similarly, the gall bladder and biliary tract can be viewed as the border between liver and intestinal tract, where many interesting things occur with profound impact on both systems. Combined efforts by researchers in the field of hepatology and gastrointestinal motility should brake down the Berlin wall of ignorance of one of the most common diseases in the Western world.

K J VAN ERPECUM G P VAN BERGE-HENEGOUWEN

Department of Gastroenterology,

University Hospital Utrecht,

Postbox 85500,

3508 GA Utrecht,

The Netherlands

Correspondence to: Dr van Erpecum.

1 Naunyn B. A treatise on cholelithiasis. New Syndenham Society, 1896.

2 Shaffer EA. Abnormalities in gall bladder function in cholesterol gallstone disease: bile and blood, mucosa and muscle-the list lengthens. Gastroenterology 1992;102:1808-12.

3 Small DM, Rapo S. The source of abnormal bile in patients with cholesterol gallstones. N Engl F Med 1970;283:53-7.

4 Pomeranz IS, Shaffer EA. Abnormal gall bladder emptying in a subgroup of patients with gallstones. Gastroenterology 1985;88:787-91.

5 Forgacs IC, Maisley MN, Murphy GM, et al. Influence of gallstones and ursodeoxycholic acid therapy on gall bladder emptying. Gastroenterology 1984;87:299-307.

6 Cabral DJ, Small DM. Physical chemistry of bile. In: Schultz SG, Forte JG, Rauner BB, eds. Handbook of physiology-The gastrointestinal system III. Section 6. Baltimore: Waverly Press, 1989:621-62.

7 van Erpecum KJ, Carey MC. Influence of bile salts on molecular interactions between sphingomyelin and cholesterol: relevance to bile formation and stability. Biochim Biophys Acta 1997;1345:269-82.

8 Carey MC. Formation and growth of gallstones: the new synthesis. In: Fromm H, Leuschner U, eds. Bile acids, cholestasis and gallstones. Dordrecht: Kluwer, 1995:147-75.

9 Holan KR, Holzbach RT, Hermann RE, et al. Nucleation time: a key factor in the pathogenesis of cholesterol gallstone disease. Gastroenterology 1979;77:611-17.

10 Halpern Z, Dudley MA, Kibe A, et al. Rapid vesicle formation and aggregation in abnormal human biles: A time-lapse video-enhanced contrast microscopy study. Gastroenterology 1986;90:875-85. 
11 van Erpecum KJ, van Berge-Henegouwen GP, Stolk MFJ, et al. Fasting gall bladder volume, postprandial emptying and cholecystokinin release in gallstone patients and normal subjects. F Hepatol 1992;14:194-202.

12 Portincasa P, Di Ciaula A, Baldassarre G, et al. Gall bladder motor function in gallstone patients: sonographic and in vitro studies on the role of gallstones, smooth muscle function and gall bladder wall inflammation. $\mathcal{F}$ Hepatol 1994;21:430-40

13 Lanzini A, Jazrawi RP, Northfield TC. Simultaneous quantitative measurements of absolute gall bladder storage and emptying during fasting and eating in humans. Gastroenterology 1987;92:852-61.

14 Howard PJ, Murphy GM, Dowling RH. Gall bladder emptying patterns in response to a normal meal in healthy subjects and patients with gallstones: ultrasound study. Gut 1991;32:1406-11.

15 Jazrawi RP, Pazzi P, Petroni ML, et al. Postprandial gall bladder motor function: refilling and turnover of bile in health and cholelithiasis. Gastroenterology 1995;109:582-91.

16 Chen Q, Amaral J, Oh S, et al. Gall bladder relaxation in patients with pigment and cholesterol stones. Gastroenterology 1997;113:930-7.

$17 \mathrm{Xu}$ QW, Shaffer EA. The potential site of impaired gall bladder contractility in an animal model of cholesterol gallstone disease. Gastroenterology 1996; 110:251-7.

18 Behar J, Rhim B, Thompson WR, et al. Inositol triphosphate restores impaired human gall bladder motility associated with cholesterol stones. Gastroenterology 1993;104:563-8.

19 Fridhandler TM, Davison JS, Shaffer EA. Defective gall bladder contractility in the ground squirrel and prairie dog during the early stages of cholesterol gallstone formation. Gastroenterology 1983;85:830-6.

20 Brugge WR, Brand DL, Atkins $\mathrm{H}$, et al. Gall bladder dyskinesia in chronic acalculous cholecystitis. Dig Dis Sci 1986;31:461-7.

21 Pauletzki J, Althaus R, Holl J, et al. Gall bladder emptying and gallstone formation: a prospective study on gallstone recurrence. Gastroenterology 1996;111:765-71.

22 Sitzmann JV, Pitt HA, Steinborn PA, et al. Cholecystokinin prevents parenteral nutrition induced biliary sludge in humans. Surg Gynecol Obstet 1990;170:25-31.

23 Gebhard RL, Prigge WF, Ansel HJ, et al. The role of gall bladder emptying in gallstone formation during diet-induced rapid weight loss. Hepatology 1996;24:544-8.

24 Shoda J, He B, Tanaka N, et al. Increase of deoxycholate in supersaturated bile of patients with cholesterol gallstone disease and its correlation with de novo syntheses of cholesterol and bile acids in liver, gall bladder emptying, and small intestinal transit. Hepatology 1995;21:1291-302.

25 Heaton KW, Emmett PM, Symes CL, et al. An explanation for gallstones in normal-weight women: slow intestinal transit. Lancet 1993;341:8-10.

26 Jörgensen T. Abdominal symptoms and gallstone disease: an epidemiological investigation. Hepatology 1989;9:856-60.

27 Thomas LA, Bathgate T, Veysey MJ, et al. Is cholelithiasis an intestinal disease [abstract]? Gut 1997;41:A2.

28 Hussaini SH, Pereira SP, Murphy GM, et al. Deoxycholic acid influences cholesterol solubilization and microcrystal nucleation time in gall bladder bile. Hepatology 1995;22:1735-44.

29 Hussaini SH, Murphy GM, Kennedy C, et al. The role of bile composition and physical chemistry in the pathogenesis of octreotide-associated gall bladder stones. Gastroenterology 1994;107:1503-13.

30 Veysey MJ, Gathercole DJ, Mallet A, et al. Large bowel transit time influences deoxycholic acid input rate and pool size-risk factors for octreotide-induced gallstones [abstract]. Gastroenterology 1997;112:A525.

31 Berr F, Kullak-Ublick G, Paumgartner G, et al. $7 \alpha$-dehydroxylating bacteria enhance deoxycholic acid input and cholesterol saturation of bile in enhance deoxycholic acid input and cholesterol saturation

$32 \mathrm{Xu}$ Q, Scott RB, Tan DTM, et al. Slow intestinal transit: a motor disorder contributing to cholesterol gallstone formation in the ground squirrel. Hepatology 1996;23:1664-72.

33 Wang DQ-H, Paigen B, Carey MC. Phenotypic characterization of Lith genes that determine susceptibility to cholesterol cholelithiasis in inbred mice: physical-chemistry of gallbladder bile. 7 Lipid Res 1997;38:1395-411.

34 van der Werf SDJ, van Berge-Henegouwen GP, Ruben AT, et al. Biliary lipids, bile acid metabolism, gall bladder motor function and small intestinal transit during ingestion of a sub-fifty oral contraceptive. f Hepatol 1987;4:318-26.
35 Vlahcevic ZR, Stravitz RT, Heuman DM, et al. Quantitative estimations of the contribution of different bile acid pathways to total bile acid synthesis in the rat. Gastroenterology 1997;113:1949-57.

36 Cooper AD. Bile salt biosynthesis: an alternate synthetic pathway joins the mainstream. Gastroenterology 1997;113:2005-8.

37 Brown HJ, Read NW, Richardson A, et al. Characteristics of lipid substances activating the ileal brake in the rat. Gut 1990;31:1126-9.

$38 \mathrm{Xu}$ Q, Shaffer EA. The influence of bile salts on small intestinal motility in the guinea pig in vitro. Gastroenterology 1992;103:29-35.

39 Stolk MFJ, van de Heijning BJM, van Erpecum KJ, et al. The effect of bile salt hydrophobicity on nucleation of several types of cholesterol crystals from model bile vesicles. $\mathcal{F}$ Hepatology 1994;20:802-10.

40 Wang DQ-H, Carey MC. Complete mapping of crystallization pathways during cholesterol precipitation from model bile: influence of physicalchemical variables of pathophysiologic significance and identification of a stable liquid-crystalline state in cold, dilute and hydrophilic bile salt-containing systems. 7 Lipid Res 1996;37:606-30.

41 Ozeki K, Sarna SK, Condon RE, et al. Enterohepatic circulation is essential for regular cycling of duodenal migrating motor complexes in dogs. Gastroenterology 1992;103:759-67.

42 Stolk MFJ, van Erpecum KJ, Smout AJPM, et al. Motor cycles with phase III in antrum are associated with high motilin levels and prolonged gall bladder emptying. Am f Physiol 1993;264:G596-600.

43 DiMagno EP, Hendricks JC, Go VLW, et al. Relationships among canine fasting pancreatic and biliary secretions, pancreatic duct pressure, and duodenal phase III motor activity_-Boldireff revisited. Dig Dis Sci 1979;24: 689-93.

44 Svenberg T, Nilsson I, Samuelson K, et al. Studies on the causal relationship between gall bladder emptying and motilin release in man. Acta Chir Scand 1984;520:S59-61.

45 Stolk MFJ, van Erpecum KJ, Samson M, et al. Interdigestive gall bladder emptying, antroduodenal motility and motilin release in cholesterol gallstone patients. In: Stolk MFJ, ed. Pathogenesis of cholesterol gallstones. Utrecht: Thesis Universiteit Utrecht, 1993:65-78.

$46 \mathrm{Xu} \mathrm{Q}-\mathrm{W}$, Scott RB, Tan DTM, et al. Altered migrating myoelectrical complex (MMC) in an animal model of cholesterol gallstone disease [abstract]. Gastroenterology 1997;112:A1417.

47 Itoh $\mathrm{Z}$, Takahashi I, Nakaya $\mathrm{M}$, et al. Interdigestive gall bladder bile concentration in relation to periodic contraction of gall bladder in the dog. Gastroenterology 1982;83:645-51.

48 van Erpecum KJ, van Berge-Henegouwen GP, Stoelwinder B, et al. Bile concentration is a key factor for nucleation of cholesterol crystals and cholesterol saturation index in gall bladder bile of gallstone patients. Hepatology 1990;11:1-6.

49 van Erpecum KJ, Stolk MFJ, van den Broek AMWC, et al. Bile concentration promotes nucleation of cholesterol monohydrate crystals by increasing the cholesterol concentration in the vesicles. Eur 7 Clin Invest 1993;23:283-8.

50 Conter RL, Roslyn JJ, Porter-Fink V, et al. Gall bladder absorption increases during early cholesterol gallstone formation. Am f Surg 1986;151:184-92.

51 Strichartz SD, Abedin MZ, Abdou MS, et al. The effects of amiloride on biliary calcium and cholesterol gallstone formation. Ann Surg 1989;209: $152-6$.

52 Ginanni Corradini S, Giovannelli L, Elisei W, et al. Cholesterol molar percent of bile influences the rate of phosphatidylcholine but not cholesterol absorption by the gall bladder mucosa [abstract]. Gut 1997;41:A32.

53 Ross PE, Butt AN, Gallacher C. Cholesterol absorption by the gall bladder. f Clin Pathol 1990;43:572-5.

54 Jacyna MR, Ross PE, Bakar MA, et al. Characteristics of cholesterol absorption by human gall bladder: relevance to cholesterolosis. $f$ Clin Pharmacol 1987;40:524-9.

55 Stolk MFJ, van Erpecum KJ, Renooij W, et al. Gall bladder emptying in vivo, bile composition and nucleation of cholesterol crystals in patients with cholesterol gallstones. Gastroenterology 1995;108:1882-8

56 Keulemans YCA, Mok KS, de Wit LT, et al. Hepatic bile versus gall bladder bile: a comparison of protein and lipid concentration and composition in cholesterol gallstone patients. Hepatology 1998;28:11-16. 